\title{
Urinary excretion of renin and angiotensinogen in hypertensive children and adolescents
}

Magdalena Zając, Agnieszka Rybi-Szumińska, Justyna Storonowicz, Piotr Protas, Anna Wasilewska

Department of Paediatrics and Nephrology, Medical University of Bialystok, Bialystok, Poland

Submitted: 2 April 2018; Accepted: 17 October 2018

Online publication: 7 October 2019

Arch Med Sci 2021; 17 (5): 1325-1331

DOI: https://doi.org/10.5114/aoms.2019.88482

Copyright $\odot 2019$ Termedia \& Banach

\section{Abstract}

Introduction: In recent years hypertension has become an emerging condition in the young population. It has been proposed that the renin-angiotensin system plays an important role in regulation of blood pressure. We assessed whether activation of the intrarenal renin-angiotensin system occurs in hypertensive children and adolescents and what better reflects its activity: urine angiotensinogen (AGT) or urine renin (REN).

Material and methods: The study was conducted on a sample of 58 subjects with primary hypertension (HT) and 29 normotensive children and adolescents. We measured urine REN and AGT excretion and assessed the values in relation to blood pressure (BP) and other clinical parameters. Both REN and AGT values were calculated by urine creatinine: REN/cr. and AGT/cr., respectively.

Results: We observed higher urine REN/cr. values in hypertensive subjects in comparison to the reference group (6.99 vs. 2.93, $p=0.003$ ). Hypertensive participants showed positive correlations between urine REN/cr. and diastolic 24-hour BP $(r=0.42, p=0.002)$ as well as between urine REN/cr. and urine AGT/cr. ( $r=0.266, p=0.044$, respectively).

Conclusions: Increased urine REN/cr. in hypertensive children and adolescents and its positive correlation with BP may indicate its important role in the pathogenesis of HT. Perhaps urine REN/cr. could be a marker of intrarenal renin-angiotensin system activity. Nevertheless, further research should be undertaken to confirm this observation.

Key words: adolescents, blood pressure, children, renin-angiotensin system, urine.

\section{Introduction}

In recent years it has been proposed that the intrarenal renin-angiotensin system (RAS) plays an important role in maintaining sodium balance and blood pressure (BP) [1, 2]. Intrarenal RAS exerts pleiotropic regulatory actions on renal haemodynamic and transport processes [3, 4]. Kidney is unique because it has all the necessary components of the RAS in different compartments of renal structures combined with their intracellular accumulation. It was found that angiotensinogen (AGT) and angiotensin II (Ang II) levels in proximal tubules were greater than could be explained from the plasma concentrations [5-7].

Lately, significant attention has been paid to the possible markers of intrarenal RAS activity, which could be measured in the urine. Navar et al.

\author{
Corresponding author: \\ Agnieszka Rybi-Szumińska \\ MD, PhD \\ Department of Paediatrics \\ and Nephrology \\ Medical University \\ of Bialystok \\ 17 Waszyngtona St \\ 15-274 Bialystok, Poland \\ Phone: +48 857450825 \\ Fax: +48 857421838 \\ E-mail: arybiszuminska@ \\ gmail.com
}


showed that the increased Ang II concentration led to stimulation of AGT expression in proximal tubule cells [8]. What is interesting, in experimental studies a positive relationship between intrarenal Ang II levels and urine AGT excretion rates was shown, indicating that urine AGT might serve as an index of intrarenal RAS activity $[9,10]$. Higher urine AGT concentration was also found in patients with hypertension (HT), which allowed urine AGT to be considered as a potential marker of intrarenal RAS activation [11, 12]. However, another study in diabetic and non-diabetic patients with $\mathrm{HT}$ revealed that urine renin (REN), and not urine AGT or aldosterone, was an important marker of renal RAS activity [13].

Because there is no consensus in the literature about which of the components of RAS better correlates with BP and available data focus on adult hypertensive patients, this study has been designed to assess urine concentration of AGT as well as REN in hypertensive children and adolescents. There is a paucity of similar investigations in the paediatric population with primary HT. We suspect that the activation of the intrarenal RAS occurs in hypertensive children, and we have tried to verify what better reflects this activity: urine AGT or REN. We also assume that some relation between intrarenal RAS activity markers and other biochemical parameters might be possible.

\section{Material and methods}

This was a study of 58 (19 girls) hypertensive children and adolescents. The participants were referred to the Department of Paediatrics and Nephrology, Medical University of Bialystok within 24 months. The subjects were sent to our unit by general practitioners after observation of elevated BP measurements in ambulatory conditions or to carry out follow-up visits. None of them was on antihypertensive therapy.

Inclusion criteria: children and adolescents aged 4-18 years, arterial HT (confirmed by ambulatory blood pressure monitoring (ABPM) and/ or three separate casual BP measurements $>95^{\text {th }}$ centile), normal concentration of thyroid-stimulating hormone (TSH) and creatinine in serum, no abnormalities in urinalysis or ultrasound examination of the abdomen, and normal electrocardiogram.

Exclusion criteria: heart failure, renal dysfunction, confirmed secondary forms of HT, diseases of thyroid or adrenal glands, diabetes mellitus, systemic inflammatory conditions, autoimmune and haematological disorders, and use of contraceptives in females.

The reference group consisted of 29 children and adolescents (19 girls), diagnosed in our Department because of nocturnal enuresis, without any pharmacological treatment, and with normal BP. Their blood and urine tests were within the normal range. Also, their family history did not reveal HT, cardiovascular or renal disease, or diabetes. Physical examination was also normal.

Written, informed consent was obtained from parents and all participants over 16 years old. The study protocol was approved by the Local Bioethics Committee, Medical University of Bialystok.

In all subjects the medical past and present history was taken from the parents. Also, careful physical examination was performed in all participants. Body weight and height were measured using a balance beam scale and a paediatric wall-mounted stadiometer. Body mass index (BMI) was calculated as weight (kilograms) divided by the square of height (metres squared). Age- and height-specific reference values for BMI were generated by the Lambda-Mu-Sigma (LMS) method [14]. The LMS values were taken from the OLAF study, published by Kułaga et al. [15]. Blood pressure was measured using an automatic manometer Mindray VS 800, validated for paediatric use in a sitting position. The cuff was selected according to the Fourth Report on the Diagnosis, Evaluation, and Treatment of High Blood Pressure in Children and Adolescents [16]. Subjects had three measurements taken in 3-minute intervals, and the average of the second and third measurement were calculated. Then estimated standard deviation score (SDS) of systolic and diastolic BP was used for further analysis. We used reference data based on the OLAF study [15].

In the hypertensive and reference group, after 12-hour overnight fasting, blood and urine samples were taken for the measurement of serum concentrations of creatinine, glucose, lipid profile, and urine creatinine as well as REN and AGT. Also, a 24-hour urine collection was carried out to assess the excretion of sodium ( $\mathrm{Na}$ ) and albumin. The estimated glomerular filtration rate (eGFR) was calculated by the Schwartz formula: eGFR $=x \times G(\mathrm{~cm}) /$ $\mathrm{Lcr}(\mathrm{mg} / \mathrm{dl})$, where $\mathrm{x}$ is a constant depending on age and sex $(0.33$ - in low birth weight infants, 0.45 - in term infants, 0.55 - in children and adolescent girls and 0.7 - in adolescent boys), $\mathrm{G}$ is height, and Lcr is the level of creatinine in the serum.

The urine concentration of REN was measured, according to the manufacturer's instructions, using a commercially available enzyme-linked immunosorbent assay (ELISA) kit (Human Renin Quantikine ELISA Kit, R\&D Systems, Minneapolis, $M N)$. In short, a monoclonal antibody specific for renin, which had been pre-coated onto a micro plate, was used to detect renin in the urine samples. After washing away any unbound substances, the antibody detecting renin, which had been conjugated to horseradish peroxidase, was added. 
Then (after the next use of wash buffer) the colour-forming peroxidase substrate containing tetramethylbenzidine was added. The colour was measured at $450 \mathrm{~nm}$ by a microtiter plate reader and compared with a standard curve. Urinary renin levels were expressed in picograms per millilitre $(\mathrm{pg} / \mathrm{ml})$. The minimum detectable concentration of REN of this assay ranged from 0.769 to $14.8 \mathrm{pg} / \mathrm{ml}$ (mean: $4.43 \mathrm{pg} / \mathrm{ml}$ ).

The urine AGT concentration was also measured using a commercially available ELISA kit (ELISA Kit for Angiotensinogen (AGT), Cloud-Clone Corp., Houston, TX). In brief, a biotin-conjugated antibody specific for AGT, which had been pre-coated onto a micro plate, was used to detect AGT in the samples of urine. Next, the biotin-binding protein conjugated to horseradish peroxidase and the colour-forming peroxidase substrate containing tetramethylbenzidine were added. The colour was measured at $450 \mathrm{~nm}$ by a microtiter plate reader and was likened with a standard curve. Urinary AGT levels were expressed in nanogram per millilitre $(\mathrm{ng} / \mathrm{ml})$. The minimum detectable concentration of AGT is typically less than $0.62 \mathrm{ng} / \mathrm{ml}$.

The REN and AGT measurements were normalised by urine concentration of creatinine to account for the influence of urinary dilution on their concentration [17]. Therefore, the concentration of REN was expressed as the urine-REN-to-creatinine ratio (renin/cr.) in picograms per milligram of creatinine (pg/mg), and the AGT concentration as the urine-AGT-to-creatinine ratio (AGT/cr.) in nanograms per milligram of creatinine $(\mathrm{ng} / \mathrm{mg})$. Serum and urine creatinine were determined by updated Jaffe reaction. Urine excretion of $\mathrm{Na}$ was assessed with a Cobas-Integra 800 analyser and Roche reagents (Roche, Indianapolis, IN). The 24-h urinary albumin excretion rate (UAER) was analysed by radioimmunoassay method. Albuminuria was considered as a 24-h UAER of $>30$ mg/day.

ABPM was performed in hypertensive children and adolescents using an oscillometric blood pressure monitor (SpaceLab, Redwood, WA). The monitors were programmed to measure BP every 15 min during the daytime (8:00-22:00) and every 30 min during the night-time (22:00-8.00), with the correction of these periods according to the individual diaries of each subject. The recording began between 8 and 9 a.m. and lasted for 24 h. Recordings with a minimum $80 \%$ of measurement and without breaks longer than $2 \mathrm{~h}$ were accepted. The mean systolic BP (SBP) and diastolic BP (DBP) were calculated for the 24-h and for the awake and asleep periods. Also, the load systolic (LSBP) and diastolic (LDBP) blood pressure during the day and night were analysed. HT on the basis of ABPM was defined as mean systolic or diastolic daytime or night-time BP levels $\geq 95^{\text {th }}$ percentile and LSBP or LDBP daytime or night-time levels $>25 \%$ [18]. The values were adjusted by gender and body height according to the reference values provided by Wühl et al. [19]. Each subject or his/ her parent was asked to record the bedtime and time of awakening. After $24 \mathrm{~h}$, the cuff and monitor were removed, and the data were downloaded using the manufacturer's software.

\section{Statistical analysis}

Sample-size calculation was done using e-picos software (AEK Contracted R\&D and Education Company, Mersin Technoscope, Turkey). Data analysis was performed using a computer program Statistica (data analysis software system, StatSoft Inc., 2012), version 10. Categorical variables are expressed as counts (percentage) and continuous variables as median and interquartile range (Q1Q3) unless stated otherwise. The Shapiro-Wilk test was used for normality of distribution. The comparison between the two groups was done with the $\chi^{2}$ test and Fisher exact test for categorical variables and Mann-Whitney test if variables were not normally distributed. Correlations between REN/cr., AGT/cr., and other variables (values and clinical and laboratory parameters) were evaluated by Spearman's test. The value of $p<0.05$ was considered statistically significant.

\section{Results}

The demographic, clinical, and laboratory data of the study and reference group are summarised in Table I. In the hypertensive group male subjects were predominant, which is consistent with other reports. The body weight and height of HT subjects was higher than the healthy children and adolescents. Nevertheless, there was no statistically significant difference between the median percentile of body weight or height between those two groups $(p>0.05)$. Similarly, BMI SDS values did not differ significantly between hypertensive and reference participants. Ten subjects from the HT group and five from reference were classified as obese. Both systolic and diastolic BP (SBP SDS and DBP SDS) were significantly higher in the HT group $(p<0.01)$. Moreover, the HT subjects had significantly higher serum concentration of creatinine $(p<0.05)$, although within the normal range for age and sex in both groups. The groups did not differ in the serum albumin, glucose, and lipid concentrations. There was also no difference in urine $\mathrm{Na}$ excretion.

When compared to the reference group, hypertensive patients had higher urine REN/cr. (6.99 vs. $2.93 \mathrm{pg} / \mathrm{mg}, p=0.003$ ) (Figure 1) but in terms of urine AGT/cr. there was no difference between 
Table I. Anthropometric, clinical, and metabolic characteristics of examined (HT) and reference (R) groups (together)

\begin{tabular}{|c|c|c|c|}
\hline Variable & HT (58) & $\mathrm{R}(29)$ & $P$-value \\
\hline Age [years] & $14.5(9-17)$ & $10(8-15)$ & 0.066 \\
\hline Height $[\mathrm{cm}]$ & $163(140-174)$ & $146(135-165)$ & 0.032 \\
\hline Weight $[\mathrm{kg}]$ & $62.5(42.4-79.5)$ & $44.5(36-60.8)$ & 0.017 \\
\hline BMI SDS & $1.65(0.81-2)$ & $1.38(0.05-1.92)$ & 0.285 \\
\hline Glucose $[\mathrm{mg} / \mathrm{dl}]$ & $91.5(86-95.5)$ & $91(86-92)$ & 0.224 \\
\hline $\mathrm{TG}[\mathrm{mg} / \mathrm{dl}]$ & $76.5(54-102)$ & $73(46-88)$ & 0.549 \\
\hline $\mathrm{HDL}[\mathrm{mg} / \mathrm{dl}]$ & $52(44-63)$ & $51(39-62)$ & 0.394 \\
\hline SBP SDS & $1.79(1.25-2.32)$ & $0.75(-0.35-0.82)$ & 0.006 \\
\hline DBP SDS & $0.54(-0.11-1.22)$ & $0.48(-0.3-0.69)$ & 0.001 \\
\hline SBP 24-h ABPM SDS & $1.79(1.25-2.32)$ & & \\
\hline DBP 24-h ABPM SDS & $0.54(-0.11-1.22)$ & & \\
\hline Serum creatinine $[\mathrm{mg} / \mathrm{dl}]$ & $0.61(0.44-0.76)$ & $0.51(0.41-0.61)$ & 0.023 \\
\hline $\mathrm{eGFR}\left[\mathrm{ml} / \mathrm{min} / 1.73 \mathrm{~m}^{2}\right]$ & $158.75(144.1-176.7)$ & $167.1(139.6-188)$ & 0.512 \\
\hline Serum Na concentration [mmol/l] & $140(139-141)$ & $139(138-141)$ & 0.202 \\
\hline Serum $\mathrm{K}$ concentration $[\mathrm{mmol} / \mathrm{l}]$ & $4.28(4.11-4.5)$ & $4.29(4.05-4.57)$ & 0.927 \\
\hline Urinary excretion of albumin [mg/day] & $3.76(2.04-8.12)$ & $3.96(1.56-12.74)$ & 0.937 \\
\hline Urinary excretion of $\mathrm{Na}[\mathrm{mg} /$ day] & $97.75(62.22-148.86)$ & $100.54(75.46-120.55)$ & 0.14 \\
\hline Urine renin/cr. [pg/mg] & $6.99(3.47-9.87)$ & $2.93(2.69-4.48)$ & 0.003 \\
\hline Urine AGT/cr. [ng/mg] & $3.19(1.68-3.91)$ & $2.89(1.49-4.59)$ & 0.544 \\
\hline Urine creatinine $[\mathrm{mg} / \mathrm{dl}]$ & $100.72(67.41-163.7)$ & $99.93(52.3-149.79)$ & 0.574 \\
\hline
\end{tabular}

$M$ - male, F-female, cm - centimetres, $\mathrm{kg}$ - kilograms, BMI - body mass index, LMS - Lambda-Mu-Sigma, SBP-systolic blood pressure, $D B P$ - diastolic blood pressure, SDS - standard deviation score, eGFR - estimated glomerular filtration rate, Na - sodium, $K$ - potassium, renin/cr. - renin-to-creatinine ratio, AGT - angiotensinogen, AGT/cr. - angiotensinogen-to-creatinine ratio, $24 \mathrm{~h}$ ABPM - 24-h ambulatory blood pressure measurement; values are presented as the median with the interquartile range $\left(Q_{1}-Q_{3}\right)$.

these two groups $(p=0.544)$. We found no differences in the urine excretion of REN and AGT between boys and girls $(p>0.05)$.

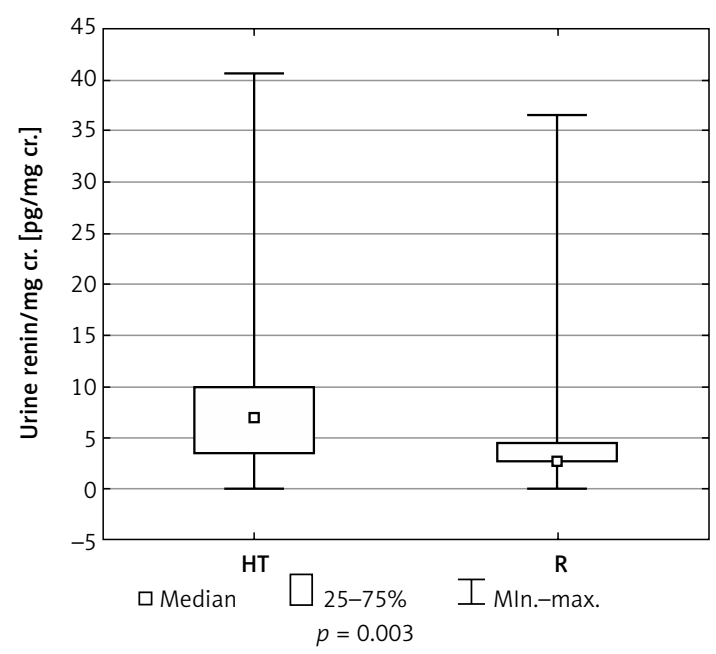

Figure 1. Urine renin/cr. in hypertensive and reference children (pg/mg), $p=0.003$
Then we analysed correlations between urine REN/cr., AGT/cr., and parameters of physical development, BP, biochemical parameters, and urine sodium and albumin excretion (Table II) in hypertensive children. Statistically significant positive correlations were found between urine REN/cr. and 24-h ABPM DBP SDS (24-h ABPM was performed only in the HT group) $(r=0.419, p=0.002)$. We did not observe a significant correlation between urine REN/cr. and casual BP. What is more, in HT children we found a positive correlation between urine REN/cr. and urine AGT/cr. $(r=0.266$, $p=0.044)$.

In the reference group we did not find any significant correlations between examined parameters.

\section{Discussion}

In our study we found that hypertensive young patients had higher urine REN/cr. when compared to their healthy peers, and this correlated positively with diastolic 24-h ABPM measurements in hypertensive children. However, in hypertensive 
participants the correlation between urine REN/cr. and casual BP was not observed.

Interestingly, we found positive correlation between urine REN/cr. and AGT/cr. in the HT group.

In our study, neither in the HT group nor in the reference group, did we find any difference in urine REN/cr. or AGT/cr. values between girls and boys, which is consistent with an earlier report in adults [13]. In patients with HT we might expect activation of intrarenal RAS, which in some previous studies seemed to be even more closely related to HT than plasma REN activity (PRA). The increase in REN production in distal tubules seemed to be independent of high BP in 2-kidney 1-clip Goldblatt hypertensive rats [20]. Also, in contrast to the reduction of PRA in response to intravenous infusion of Ang II, the increase of REN in distal nephron was observed in Ang II-dependent hypertensive rats [21]. It looks similar in a transgenic mouse model [22]. Also, in hypertensive patients the activation of the intrarenal RAS was observed [23-26] but without the simultaneous increase in PRA. These observations indicate that the systemic and local RAS may be regulated in different ways and, what is more, local RAS activity may be even more closely related to HT.

The main question is: what reflects tissue RAS activity the best? Based on our observations we suggest that REN is this marker.

To the best of our knowledge, this study is the first that presents a significant positive association between urine REN and BP in hypertensive children and adolescents.

Angiotensin is too unstable to be measured in the urine. Most available literature findings focus on AGT as a good indicator of RAS activation $[27,28]$. However, our study reveals that urine REN may be a more important marker of renal RAS activity. We did not find any important difference in urine AGT/cr. between normo- and hypertensive subjects, contrary to urine REN/cr., which was significantly higher in patients with HT. Nevertheless, some correlation between urine REN/cr. and urine AGT/cr. was observed. Urine REN/cr. correlated with diastolic 24-h ABPM measurements in hypertensive participants. 24-h ABPM is a gold standard of diagnosing HT. Elevation of systolic BP may result from many different factors like stress, physical activity, etc. Correlation between urine REN/cr. and diastolic 24-h ABPM might indicate its engagement in $\mathrm{HT}$ pathogenesis.

Although many reports suggest that AGT is an important compound of the renal RAS, REN seems to be a better indicator of this activity. This effect may be related to the fact that AGT urine excretion mimics urine albumin and total protein excretion, while REN is not similar to any other substances
Table II. Correlations between urine renin/cr. and other physical parameters and biochemical markers in serum and urine in the HT group

\begin{tabular}{|lcc|}
\hline Variable & $\begin{array}{c}\text { Correlation } \\
\text { coefficient }(r)\end{array}$ & $P$-value \\
\hline BMI (LMS) & 0.153 & 0.0284 \\
\hline Glucose [mg/dl] & 0.232 & 0.067 \\
\hline TG [mg/dl] & 0.04 & 0.759 \\
\hline HDL [mg/dl] & 0.005 & 0.973 \\
\hline SBP SDS & 0.159 & 0.328 \\
\hline DBP SDS & 0.185 & 0.258 \\
\hline 24-h ABPM SBP SDS & 0.186 & 0.174 \\
\hline 24-h ABPM DBP SDS & 0.419 & 0.002 \\
\hline $\begin{array}{l}\text { Urinary excretion of Na } \\
\text { [mg/dl] }\end{array}$ & -0.212 & 0.12 \\
\hline $\begin{array}{l}\text { Urinary excretion of } \\
\text { albumin [mg/24 h] }\end{array}$ & -0.046 & 0.734 \\
\hline $\begin{array}{l}\text { Urinary AGT/cr. [ng/mg] } \\
\text { BM/ - body mass index, LMS - Lambda-Mu-Sigma }\end{array}$ & 0.266 & 0.044 \\
\hline
\end{tabular}

$B M I$ - body mass index, LMS - Lambda-Mu-Sigma, TG - triglycerides, HDL - high-density lipoprotein, SBP - systolic blood pressure, DBP - diastolic blood pressure, SDS - standard deviation score, Na - sodium, AGT - angiotensinogen, AGT/cr. angiotensinogen-to-creatinine ratio.

secreted in urine [13]. All the above findings are consistent with the observations of van den Heuvel et al., who also demonstrated that urine REN is the most important marker of renal RAS activity in diabetic patients [13].

It is known that Ang II stimulates sodium reabsorption in distal nephron activating the Ang II receptors, type 1 (AT1 receptors). We found a negative correlation between urine renin/cr. and urinary excretion of $\mathrm{Na}$, which confirms this dependence. However, this correlation was on the border of statistical significance.

We should mention the limitations of this study. First of all, it was carried out on a relatively small sample of individuals and was limited to a single-centre patient population. Unfortunately, the examined groups were not sex-matched; however, in our opinion it did not influence the results because urine REN/cr. and urine AGT/cr. levels did not differ between males and females. In the HT group we observed higher incidence of obesity; however, SDS BMI did not differ significantly between the two groups, and the possible influence of body weight on the results of this study is probably minimal. We examined only patients with primary HT and did not compare those findings to patients with secondary HT. We also did not assess the influence of antihypertensive therapy (especially with angiotensin-converting-enzyme inhibitors) on the local RAS activity.

The pathogenesis of essential HT in the paediatric population is still not well known. There 
are broad studies in the field involving not only REN or AGT but also new endothelial proteins like multidrug-resistant protein-1 (MRP1), playing a crucial role in the hypertensive response to Ang II $[29,30]$.

In conclusion, these findings, although preliminary, suggest that intrarenal RAS may be one of the key players in the pathogenesis of HT in young patients. What is more, we conclude that urine REN better reflects intrarenal RAS activity than urine AGT. Nevertheless, further research on a much larger, sex-matched group of hypertensive subjects should be undertaken. Additionally, the influence of ACE inhibitors on urine REN and AGT would probably deliver some interesting information.

\section{Acknowledgments}

The study protocol was approved by the Local Committee of Bioethics, Medical University of Bialystok.

\section{Conflict of interest}

The authors declare no conflict of interest.

\section{References}

1. Ingert C, Grima M, Coquard C, Barthelmebs M, Imbs JL. Contribution of angiotensin II internalization to intrarenal angiotensin II levels in rats. Am J Physiol Renal Physiol 2002; 283: F1003-10.

2. Shao W, Seth DM, Prieto MC, Kobori H, Navar LG. Activation of the renin-angiotensin system by a low-salt diet does not augment intratubular angiotensinogen and angiotensin II in rats. Am J Physiol Renal Physiol 2013; 304: F505-14.

3. Kobori H, Nangaku M, Navar LG, Nishiyama A. The intrarenal renin-angiotensin system: from physiology to the pathobiology of hypertension and kidney disease. Pharmacol Rev 2007; 59: 251-87.

4. Navar LG, Prieto MC, Satou R, Kobori H. Intrarenal angiotensin II and its contribution to the genesis of chronic hypertension. Curr Opin Pharmacol 2011; 11: 180-6.

5. Von Thun AM, Vari RC, el-Dahr SS, Navar LG. Augmentation of intrarenal angiotensin II levels by chronic angiotensin II infusion. Am J Physiol 1994; 266: F120-8.

6. Zou LX, Hymel A, Imig JD, Navar LG. Renal accumulation of circulating angiotensin II in angiotensin II-infused rats. Hypertension 1996; 27: 658-62.

7. Navar LG, Harrison-Bernard LM, Nishiyama A, Kobori H. Regulation of intrarenal angiotensin II in hypertension. Hypertension 2002; 39: 316-22.

8. Navar LG, Harrison-Bernard LM, Imig JD, Wang CT, Cervenka L, Mitchell KD. Intrarenal angiotensin II generation and renal effects of AT1 receptor blockade. J Am Soc Nephrol 1999; 10: S266-72.

9. Kobori H, Harrison-Bernard LM, Navar LG. Urinary excretion of angiotensinogen reflects intrarenal angiotensinogen production. Kidney Int 2002; 61: 579-85.

10. Kobori H, Nishiyama A, Harrison-Bernard LM, Navar LG. Urinary angiotensinogen as an indicator of intrarenal Angiotensin status in hypertension. Hypertension 2003; 41: 42-9.
11. Michel FS, Norton GR, Maseko MJ, Majane OH, Sareli P, Woodiwiss AJ. Urinary angiotensinogen excretion is associated with blood pressure independent of the circulating renin-angiotensin system in a group of African ancestry. Hypertension 2014; 64: 149-56.

12. Kobori $H$, Alper AB Jr, Shenava R, et al. Urinary angiotensinogen as a novel biomarker of the intrarenal renin-angiotensin system status in hypertensive patients. Hypertension 2009; 53: 344-50.

13. van den Heuvel $M$, Batenburg WW, Jainandunsing $S$, et al. Urinary renin, but not angiotensinogen or aldosterone, reflects the renal renin-angiotensin-aldosterone system activity and the efficacy of renin-angiotensin-aldosterone system blockade in the kidney. J Hypertens 2011; 29: 2147-55.

14. Cole TJ, Green PJ. Smoothing reference centile curves: the LMS method and penalized likelihood. Stat Med 1992; 11: 1305-19.

15. Kułaga Z, Litwin M, Tkaczyk M, et al. Polish 2010 growth references for school-aged children and adolescents. Eur J Pediatr 2011; 170: 599-609.

16. Falkner B, Daniels SR. Summary of the Fourth Report on the diagnosis, evaluation, and treatment of high blood pressure in children and adolescents. Hypertension 2004; 44: 387-8.

17. Garde AH, Hansen AM, Kristiansen J, Knudsen LE. Comparison of uncertaintnies related to standardization of urine samples with volume and creatinine concentration. Ann Occup Hyg 2004; 48: 171-9.

18. Flynn JT, Daniels SR, Hayman LL, et al. American Heart Association Atherosclerosis, Hypertension and Obesity in Youth Committee of the Council on Cardiovascular Disease in the Young. Update: ambulatory blood pressure monitoring in children and adolescents: a scientific statement from the American Heart Association. Hypertension 2014; 63: 1116-35.

19. Wühl E, Witte K, Soergel M, Mehls O, Schaefer F; German Working Group on Pediatric Hypertension. Distribution of 24-h ambulatory blood pressure in children: normalized reference values and role of body dimensions. J Hypertens 2002; 20: 1995-2007.

20. Liu L, Gonzalez AA, McCormack M, et al. Increased renin excretion is associated with augmented urinary angiotensin II levels in chronic angiotensin II-infused hypertensive rats. Am J Physiol Renal Physiol 2011; 301: F1195-201.

21. Sachetelli S, Liu Q, Zhang SL, et al. RAS blockade decreases blood ressure and proteinuria in transgenic mice overexpressing rat angiotensinogen gene in the kidney. Kidney Int 2006; 69: 1016-23.

22. Lavoie JL, Lake-Bruse KD, Sigmund CD. Increased blood pressure in transgenic mice expressing both human renin and angiotensinogen in the renal proximal tubule. Am J Physiol Renal Physiol 2004; 286: F965-71.

23. Perlstein TS, Gumieniak O, Hopkins PN, et al. Uric acid and the state of the intrarenal renin-angiotensin system in humans. Kidney Int 2004; 66: 1465-70.

24. Admiraal PJ, Derkx FH, Danser AH, Pieterman $H$, Schalekamp MA. Intrarenal de novo production of angiotensin I in subjects with renal artery stenosis. Hypertension 1990; 16: 555-63.

25. Admiraal PJ, Danser AH, Jong MS, Pieterman H, Derkx FH, Schalekamp MA. Regional angiotensin II production in essential hypertension and renal artery stenosis. Hypertension 1993; 21: 173-84. 
26. Lai KN, Leung JC, Lai KB, To WY, Yeung VT, Lai FM. Gene expression of the renin-angiotensin system in human kidney. J Hypertens 1998; 16: 91-102.

27. Kuroczycka-Saniutycz E, Wasilewska A, Sulik A, Milewski R. Urinary angiotensinogen as a marker of intrarenal angiotensin II activity in adolescents with primary hypertension. Pediatr Nephrol 2013; 28: 1113-9.

28. Urushihara M, Kondo S, Kagami S, Kobori H. Urinary angiotensinogen accurately reflects intrarenal renin-angiotensin system activity. Am J Nephrol 2010; 31: 318-32.

29. Widder J, Guzik T, Mueller CFH, et al. Role of the multidrug resistance protein-1 in hypertension and vascular dysfunction caused by angiotensin II. Arterioscler Thromb Vasc Biol 2007; 27: 762-8.

30. Jehle J, Müller CFH, Aksoy A, Zimmer S, Nickenig G, Tiyerili $V$. Genetic disruption of multidrug resistance-associated protein 1 improves endothelial function and attenuates atherosclerosis in MRP1(-/-) LDLr(-/-) double knockout mice. Arch Med Sci 2017; 13: 930-6. 